\title{
Noninvasive monitoring intracranial pressure - A review of available modalities
}

\author{
Marium Naveed Khan \\ Aga Khan University \\ Hussain Shallwani \\ University of Buffalo Neurosurgery, Buffalo, New York, USA. \\ Muhammad Ulusyar Khan \\ Aga Khan University \\ Muhammad Shahzad Shamim \\ Aga Khan University, shahzad.shamim@aku.edu
}

Follow this and additional works at: https://ecommons.aku.edu/pakistan_fhs_mc_surg_neurosurg

Part of the Life Sciences Commons, Neurology Commons, Neurosurgery Commons, and the Surgery Commons

\section{Recommended Citation}

Khan, M. N., Shallwani, H., Khan, M. U., Shamim, M. S. (2017). Noninvasive monitoring intracranial pressure - A review of available modalities. Surgical Neurology International, 8, 51.

Available at: https://ecommons.aku.edu/pakistan_fhs_mc_surg_neurosurg/147 




Surg Neurol Int. 2017; 8: 51.

PMCID: PMC5402331

Published online 2017 Apr 5. doi: $10.4103 /$ sni.sni $403 \quad 16$

PMID: $\underline{28480113}$

\title{
Noninvasive monitoring intracranial pressure - A review of available modalities
}

\author{
Marium Naveed Khan, Hussain Shallwani, ${ }^{1}$ Muhammad Ulusyar Khan, ${ }^{2}$ and Muhammad Shahzad Shamim ${ }^{3,{ }^{*}}$ \\ Aga Khan University, Aga Khan University Hospital, Karachi, Pakistan

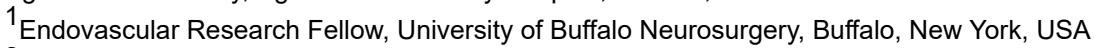 \\ 2 Division of Biological and Biomedical Sciences, Aga Khan University Hospital, Karachi, Pakistan \\ ${ }^{3}$ Division of Neurosurgery, Aga Khan University Hospital, Karachi, Pakistan \\ Marium Naveed Khan: marium.naveed@hotmail.com; Hussain Shallwani: hshallwani@ubns.com; Muhammad Ulusyar Khan: \\ muhammd.ulusyar@gmail.com; Muhammad Shahzad Shamim: shahzad.shamim@aku.edu \\ * Corresponding author
}

Received 2016 Oct 12; Accepted 2017 Jan 26.

Copyright : @ 2017 Surgical Neurology International

This is an open access article distributed under the terms of the Creative Commons Attribution-NonCommercial-ShareAlike 3.0 License, which allows others to remix, tweak, and build upon the work non-commercially, as long as the author is credited and the new creations are licensed under the identical terms.

This article has been cited by other articles in PMC.

\section{Background:}

Go to:

Intracranial pressure (ICP) monitoring is important in many neurosurgical and neurological patients. The gold standard for monitoring ICP, however, is via an invasive procedure resulting in the placement of an intraventricular catheter, which is associated with many risks. Several noninvasive ICP monitoring techniques have been examined with the hope to replace the invasive techniques. The goal of this paper is to provide an overview of all modalities that have been used for noninvasive ICP monitoring to date.

\section{Methods:}

A thorough literature search was conducted on PubMed, selected articles were reviewed in completion, and pertinent data was included in the review.

\section{Results:}

A total of 94 publications were reviewed, and we found that over the past few decades clinicians have attempted to use a number of modalities to monitor ICP noninvasively.

\section{Conclusion:}

Although the intraventricular catheter remains the gold standard for monitoring ICP, several noninvasive modalities that can be used in settings when invasive monitoring is not possible are also 
available. In our opinion, measurement of optic nerve sheath diameter and pupillometry are the two modalities which may prove to be valid options for centers not performing invasive ICP monitoring.

Keywords: Intracranial pressure, intracranial pressure monitoring, noninvasive

Intracranial pressure (ICP) is defined as the pressure inside the skull, and therefore, the pressure inside the brain tissue and the cerebrospinal fluid (CSF). The relationship between CSF and intracranial blood volumes is described by the Monroe Kellie doctrine; because the brain is incompressible, when the skull is intact, the sum of the volumes of brain, CSF, and intracranial blood is constant.[57,64] Normal ICP is usually considered to be $5-15 \mathrm{mmHg}$ in a healthy supine adult, $3-7 \mathrm{mmHg}$ in children, and 1.5$6 \mathrm{mmHg}$ in infants. $[\underline{34}, \underline{35}, \underline{64}] \mathrm{ICP}>20 \mathrm{mmHg}$ is considered to be elevated, and this is considered an important cause of secondary injury leading to irreversible brain injury and death.[499,64] ICP monitoring is used in a number of conditions; traumatic brain injury, intracerebral hemorrhage, subarachnoid hemorrhage, hydrocephalus, malignant infarction, cerebral edema, CNS infections, hepatic encephalopathy, to name a few, and in all of these conditions ICP monitoring in the light of other parameters can influence management for better outcomes.[푸, $\underline{64}, \underline{76}]$

There are several conditions where it is important to monitor ICP, as even minor fluctuations may require a change in management. The gold standard for monitoring ICP is an intraventricular catheter connected to an external pressure transducer; the catheter is placed into one of the ventricles through a burr hole.[499, 64, 76] The catheter can also be used for therapeutic CSF drainage and for administration of drugs.[49] Even though it remains an accurate and cost-effective method of ICP monitoring, it is associated with a number of complications. These include risk of infection, hemorrhage, obstruction, difficulty in placement, malposition, etc. $[\underline{49}, \underline{64}]$ Other invasive modalities for ICP monitoring, all of which entail the same complications as intraventricular catheter insertion, include intraparenchymal monitors, subdural, and epidural devices, as well as lumbar puncture measurements.[49]

Due to the number of complications associated with invasive ICP monitoring, researchers and clinicians have been trying to develop a reliable noninvasive modality for ICP monitoring. From the use of the Fontogram in the 1970s, to the ongoing experiments on acoustoelasticity effects on ICP, there is still no noninvasive ICP monitoring modality available to replace the invasive techniques.

The aim of this review is to combine a thorough search of all the available noninvasive modalities that have been used to monitor ICP, and to evaluate the feasibility and usefulness of these modalities based on existing literature.

\section{MATERIALS AND METHODS}

Go to:

A comprehensive literature search for this review was conducted on PubMed. The search was conducted from November 2014 through to February 2015, and there were no limitations on date, type, or language of the publication. The first search was conducted using the term "non invasive intracranial pressure monitoring," followed by combination of terms ("intracranial pressure"/"ICP" OR “intracranial pressure monitoring"/'ICP monitoring") AND ("non-invasive" OR "noninvasive"). These searches provided us with a total of 216 titles. The titles and abstracts were reviewed and 91 publications were selected, based on relevance to our research title, to be reviewed in detail. After reviewing these articles, a list of the noninvasive modalities available to monitor ICP was made, as shown in Table 1, which also includes the final number of articles reviewed for each modality. 
List of modalities and the number of articles reviewed for each

\begin{tabular}{lr} 
Modality & Number of \\
\hline Anterior Fontanelle Pressure & 8 \\
Skull Elasticity & 1 \\
Tympanic Membrane Displacement & 2 \\
Tissue Resonance Analysis & 1 \\
Transcranial Doppler & 8 \\
Acoustoelasticity & 3 \\
Venous Ophthalmodynamometry & 3 \\
Optic Nerve Sheath Diameter & 6 \\
Distortion-Product Otoacoustic Emissions & 12 \\
Magnetic Resonance Imaging & 57 \\
Computed Topography & 33 \\
Electroencephalography & 13 \\
Ophthalmoscopy & 7 \\
Pupillometry & 4 \\
Near Infrared Spectroscopy & 2
\end{tabular}

196

Open in a separate window

The search was then modified to include combinations of "ICP monitoring" or "noninvasive ICP monitoring" AND "anterior fontanelle pressure," "CT," "CT scan," "MRI," "optic nerve sheath diameter," "venous ophthalmodynamometry," "skull elasticity," "tissue resonance analysis," "distortion product otoacoustic emissions," "DPOAE," "otoacoustic emissions," "EEG," "electroencephalography," "optic disc evaluation," "ophthalmoscopy," "papilledema," "fundoscopy," "pupillometry," "neurological pupil index," and "near infrared spectroscopy."

The total number of titles and abstracts reviewed after these searches was 5104, not accounting for overlap present in the searches. A total of 196 publications were selected and thoroughly reviewed and read in completion. Our article included relevant data from a total of 94 publications. The length of this paper prevented us from including the role of CT and MRI in monitoring ICP. Figure 1 shows the methodology of selection. 

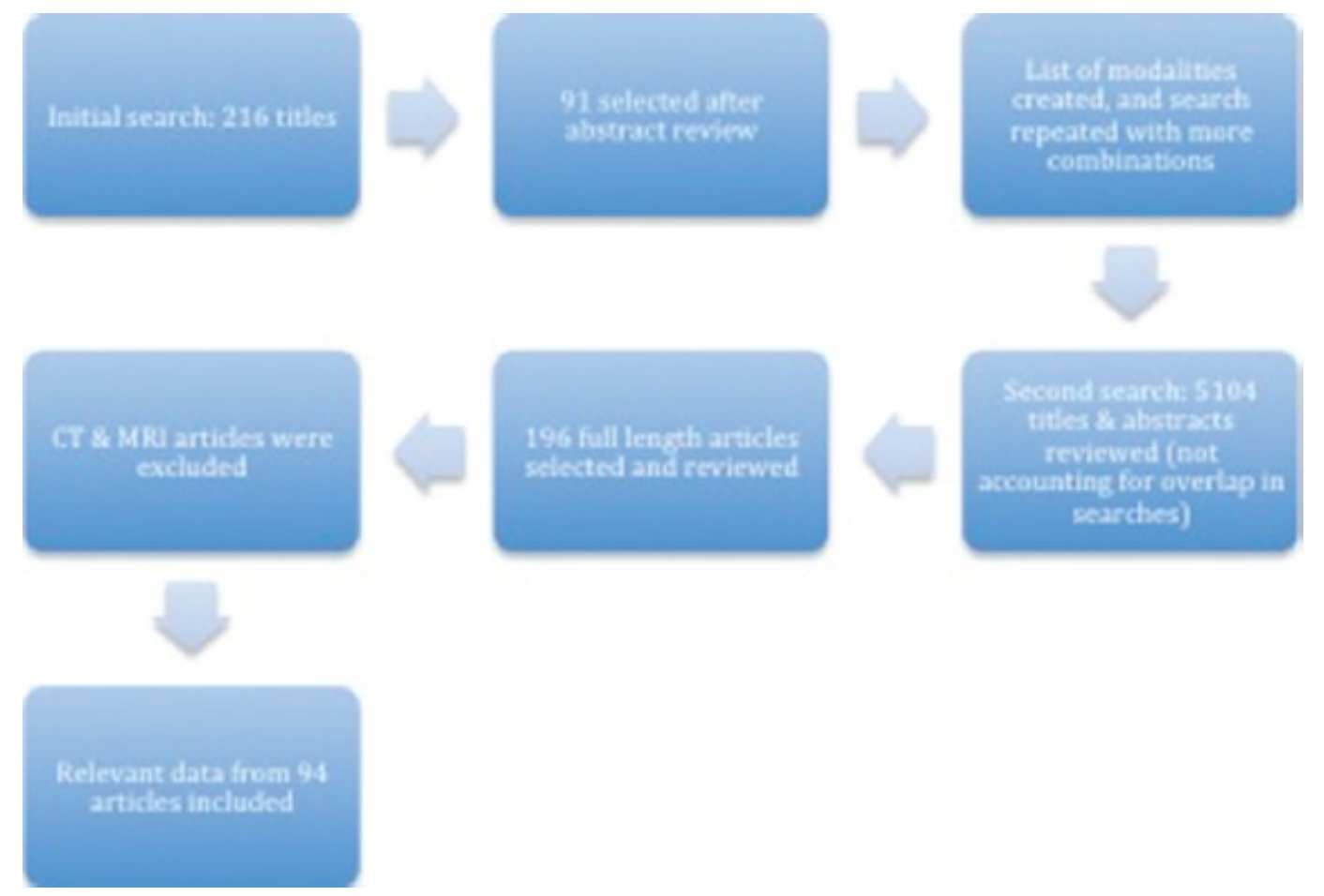

\section{Figure 1}

Process of selection of final articles

\section{Noninvasive intracranial pressure monitoring modalities}

Anterior fontanelle pressure monitoring The anterior fontanelle of the human infant is open, making it an available site to measure ICP in an infant. Many studies were conducted in the 1970s and 1980s to investigate the correlation between pressure application on the anterior fontanelle and the ICP. $[14, \underline{41}, \underline{73}, \underline{84}]$ In all of these studies, however, fixation of the device was a major issue. The methods used to fix or stabilize the device could cause deviations in the measured ICP, and interuser application force also caused variations in ICP measurements.[60] In light of these problems, the use of a device known as the Rotterdam teletransducer proved to be a suitable alternative.[20]

On the same lines, Salmon et al.[73] studied the use of an applanation transducer (called the fontogram). Laboratory and clinical studies were carried out, and it was found that the pressures recorded by the fontogram corresponded to direct measurements of ICP through an invasive catheter; the correlation coefficient was 0.98 and $P$ value $<0.001$, indicating a very good correlation. It was concluded that it was accurate to use fontanelle pressure and ICP interchangeably.

Vidyasagar et al.[프] investigated the value of measuring the anterior fontanelle pressure (AFP) in assessing ICP using the Ladd Intracranial Pressure Monitoring Device (Model 1700). The device had three components: (a) a transducer with a pressure sensitive membrane with a mirror on its surface, (b) a light transmitting ICP monitor, and (c) a pen recorder.[84] It worked on the principle that, when there is pressure on the transducer, the mirror tilts and light is reflected unevenly to the ICP monitor; the monitor then works to equalize the pressure to bring the mirror back to its original position. [84] Using this principle, Vidyasagar et al. [84] placed the transducer over the anterior fontanelle to assess ICP changes. They stated that there is a significant correlation of CSF pressure with AFP (correlation coefficient $=0.95, P<0.01$ ), indicating that the AFP was representative of ICP. 
Horbar et al.[41] also studied the effect of Ladd sensor application force on ICP measurements in infants. The Ladd M1000 ICP monitor was placed over the infant's fontanelle and then investigated by placing a device that applied a known force to the sensor. The monitor readings were shown to vary with the application force for each subject, and it was concluded that readings obtained depended on the force with which the sensor is applied to the anterior fontanelle, indicating that there could be interuser variability.

Later, Bunegin et al.[14] developed a pneumoelectronic switch to also measure the ICP through the anterior fontanelle. Studies were conducted using a newly developed infant cranial model, and subsequently healthy infants, using the Bunegin-Ablin pneumoelectronic switch as the pressure sensor. External pressure application would cause the switch to close, activating the pneumatic system, creating internal pressurization until internal and external pressures are balanced, and the balance pressure was displayed on a monitor. ICP was also measured using an indwelling ventricular catheter. The investigations concluded that the new AFP monitor was able to achieve a highly significant correlation ( $r=0.962$, confidence level $>95 \%$ ) between AFP and ICP.

A more popular method to measure AFP was via the Rotterdam teletransducer (RTT). The RTT is an implantable telemetric device that was introduced by De Jong et al. in 1979 to measure epidural pressure.[19] It was proposed that, in infants, because the anterior fontanelle is open, the skin above the fontanelle was the main difference between measuring epidural pressure and measuring AFP, and thus, the RTT could be used as a noninvasive device.[20, $\underline{60}]$ A special lightweight perspex skull adapter was designed for a controlled depth setting to account for any surface tension.[62] The transducer was fitted in the adapter, and a soft silicon fixation frame was used to place the device on the child's head. Once the device was fixed, the child could move around freely without affecting the AFP measurements.[62] Pressure depth curves (PDC) were produced and the ICP levels were determined from the plateaus; an average of at least three PDC plateaus was needed to determine the ICP level and to eliminate any interobserver variations.[60] From November 1982 till March 1988, Overweg-Plandsoen et al.[60] performed AFP measurements using the RTT in 141 patients. AFP measurements via the RTT were compared to ICP values obtained via lumbar puncture in 13 of these patients.[60] They found that the difference was less than $1 \mathrm{mmHg}$, and stated that the difference is not significant as $1 \mathrm{mmHg}$ is an acceptable measurement error.[60] They concluded that reliable, continuous, noninvasive ICP monitoring in infants was possible with the RTT placed in the adapter.

We could neither find any current use of the RTT in monitoring ICP in infants nor any of the other devices for AFP measurements. Moreover, in 2007, Wiegand et al.[91] reviewed current methods of measuring ICP in infants and stated that it could not be measured by noninvasive modalities.

Skull elasticity Attempts have been made to derive ICP from the mechanical properties of the skull bones. This is based on the hypothesis that, because the skull is not completely rigid, changes in ICP result in a small, but measurable, expansion of the skull.

In 1985, Pitlyk et al.[61] devised a noninvasive instrument for measuring skull diameter changes with changes in ICP and tested it on cadavers and dogs. Their study concluded in sufficiently high instrument sensitivity, suggesting that the device should be used in studies for clinical evaluation.

However, we could not find any studies that were conducted to assess the use of the device for clinical evaluation.

In 2009, Yue and Wang[93] studied the deformation of the skull bones as a result of changing ICP by placing strain foils on the parietal surface. The ICP variations were then recorded via the strain foils. Their results showed that the human skulls were deformed by changes in the ICP, and that the strains of the skull coincided with ICP variation. Even though these studies show a positive correlation between skull elasticity and ICP, no methods of monitoring ICP through studying the elasticity of the skull, to our knowledge, have been verified so far. 
Optic nerve sheath diameter The optic nerve sheath, which is continuous with the dura matter of the brain, is surrounded by the subarachnoid space containing CSF.[42] Therefore, when there is an increase in the pressure of the CSF, the sheath can expand.[31] In 1964, Hayreh et al.[39] showed that, due to the communication of the subarachnoid space with the intracranial cavity, changes in CSF pressure could be transmitted along the optic nerve. The optic nerve sheath diameter (ONSD) expansion can be compared to papilledema (edema of the optic disc), but unlike papilledema, ONSD expansion occurs within seconds of an acute rise of ICP.[31]

On this basis, ocular sonography has been used to measure the changes in ONSD to detect raised ICP, and it has been clinically shown that millimetric increases in the sonographic ONSD corresponds to increased ICP.[31] Several studies have been conducted to demonstrate the relationship between ONSD and increased ICP. Most studies encouraged the clinical use of sonography for ONSD in the detection of steady changes in ICP, and for screening purposes, but do not consider it as a replacement for invasive ICP monitoring.[리, $\underline{43}, \underline{44}, \underline{48}, \underline{49}, \underline{64}]$

Recent studies have shown that an increase of the ONSD in an estimated range between 4.5 and 5.5 $\mathrm{mm}$ is associated with an increased ICP $(>20 \mathrm{mmHg}) \cdot[\underline{4}, \underline{31}, \underline{32}, \underline{44}, \underline{48}, \underline{49}, \underline{56}, \underline{68}, \underline{77}, \underline{78}, \underline{81}]$ The largest of these studies to date was conducted by Rajajee et al.[67] Their study included 65 patients on whom a total of 536 ONSD measurements were performed. They concluded that an ONSD of $>4.8 \mathrm{~mm}$ corresponded to an ICP $>20 \mathrm{mmHg}$ with a sensitivity of $96 \%$ and a specificity of $94 \%$.[67] Another study, however, concluded that sonographic ONSD measurements were not reliable to monitor ICP due to poor accuracy and correlation on the basis of $36 \%$ sensitivity and $38 \%$ specificity.[으]

Sonographic ONSD measurement is a quick, efficient, and easy to learn modality for the monitoring increased ICP. However, it is important to mention the limitations associated with the measurement of the ONSD as well. Several conditions, including tumors, inflammation, sarcoidosis, and Grave's disease can possibly affect the ONSD, and it is impossible to measure ONSD in those patients with lesions of the orbit or of the optic nerve.[64] In conclusion, even though ONSD measurement cannot replace invasive ICP monitoring, it can differentiate between normal and raised ICP. Therefore, it can potentially be used for screening purposes when invasive modalities are unavailable.[64]

Venous ophthalmodynamometry The central retinal vein (CRV) passes through the optic nerve, which as described before is surrounded by CSF, resulting in both the optic nerve and the CRV to be affected by changes in ICP. Therefore, the pressure within the CRV must be as high or higher than the ICP.[26]

In 1925, Baurmann[] ] was the first to suggest that the pressure of the CRV was dependent on the ICP, and variations could be monitored by the pulsations of the CRV. When the CRV collapses, it indicates that IOP $>\mathrm{CRV}$ pressure, when it pulsates IOP $\approx \mathrm{CRV}$ pressure, and when it is clearly visible and there are no pulsations CRV pressure $>$ IOP.[27]

Baurmann[] suggested recording the CRV pressure to monitor ICP. In 2000, Firshing et al.[27] stated that his idea had not as yet been verified, and they performed a study to examine the use of venous ophthalmodynamometry in monitoring ICP noninvasively. In their study, CRV pressure of 22 patients was correlated with their ICP (which was invasively monitored). They found a high correlation ( $r=$ $0.983, P<0.001$ ), and concluded that ophthalmodynamometry could be used for momentary assessment of ICP but not for continuous assessment.

Querfurth et al.[ㅎ3] using a novel digital and portable ophthalmometer measured the venous outflow pressure (VOP) of the CRV in 6 intensive care patients and correlated it with invasive ICP readings. They found that as the ICP increased so did the VOP, the relationship was linear $(r=0.83, P<0.001)$. [흐]

In 2011 Firsching et al.[26] recorded the CRV pressure in 102 patients who also had simultaneous invasive ICP monitoring. They used an "ODM Saugnapf Dynamometer" to increase ocular pressure in 
a stepwise manner until the CRV collapsed. The use of a conversion chart helped determine the actual pressure at which the vein collapsed, this pressure was considered equal to the CRV pressure (VOP). These pressure values were then correlated with ICP values. A significant association was found between ICP and VOP $(P<0.001$ and $r=0.69)$. VOP $>30 \mathrm{mmHg}$ indicated ICP $>15 \mathrm{mmHg}$ with a probability of $84.2 \%$, and VOP $\leq 30 \mathrm{mmHg}$ indicated ICP $\leq 15 \mathrm{mmHg}$ with a probability of $92.8 \%$. The study had a sensitivity of $72.7 \%$ and specificity of $96.2 \%$. The author's concluded that venous ophthalmodynamometry is a valuable method to assess ICP noninvasively, however, it would not replace invasive procedures as it is not helpful for continuous ICP monitoring.[26]

Like other ophthalmological techniques of monitoring ICP, venous ophthalmodynamometry is a valuable technique to use for screening patients suspected to have increased ICP before carrying out an invasive technique. The method cannot replace invasive techniques, although may be used as a followup investigation in some patients.[르, $\underline{27}]$

Tympanic membrane displacement Tympanic membrane displacement (TMD) was the first audiologic method studied to monitor ICP noninvasively.[49] The TMD is measured in response to sound stimulation (acoustic reflex).[53] The stimulation results in the contraction of the stapedius muscle, resulting in the movement of the stapes. Due to the close relationship between the stapes and the oval window, cochlear fluid pressures, and therefore ICP, determine the position of the stapes. Thus, pressure changes result in movement of the stapes and the displacement of the tympanic membrane. In 1990, Reid et al.[69] compared TMD values with invasive ICP values. They investigated the effect of changes in ICP with TMD in 58 patients, and found differences in the TMD between patients with raised and normal ICP. They concluded that although an absolute ICP value could not be obtained by this method, TMD is useful in assessing raised or normal ICP.

Samuel et al.[74] prospectively studied the accuracy and repeatability of the TMD test in evaluating the ICP in 8 children with shunted hydrocephalus. They found that the TMD test had a sensitivity of $83 \%$ and specificity of $100 \%$, a positive predictive value of $100 \%$, and a negative predictive value of $29 \%$. Their results led them to the conclusion that the TMD test could be used as an investigative tool regularly in the assessment of such patients, thereby reducing the need for invasive monitoring of the ICP.[74]

Stettin et al.[79] investigated whether measuring infrasonic emissions from the tympanic membrane could monitor ICP in 31 individuals (17 patients, 14 healthy). Invasive ICP monitoring was indicated in all patients and was done so with implanted pressure sensors. An increase in ICP was stimulated by postural changes using a tilting table.[79] The infrasonic emissions were measured by recording the TMD using a probe placed in the external auditory meatus in an airtight manner.[30] It was observed that the stimulated changes in ICP were detected in waveforms both noninvasively and invasively. Absolute values of the ICP, however, could not be established. Stettin et al.,[79] therefore, concluded that infrasonic emissions could be suitable both as a screening tool and for continuous ICP monitoring. They also suggested that a possible approach for establishing absolute IC $P$ values could be done by creating a computer software that could evaluate the waveforms and peaks.[79]

A recent review article[49] suggested that, if a baseline ICP has been established, then repeated TMD measurements could be used to find a change in the ICP. There are, however, certain limitations to the TMD test. Three essential criteria are required to perform a TMD test; patent cochlear aqueduct, normal middle ear pressure, and intact stapedial reflex.[74]

Tissue resonance analysis In 2002, Michaeli et al.[55] studied the use of tissue resonance analysis (TRA) on noninvasive intracranial pressure. Their study was done on the basis of mechanical tissue vibration, which allowed them to get several resonance peaks. With each heartbeat, the vibratory and mechanical resonance of each organ and tissue vary.[55] It was found that ICP was dependent on the value of a dominant secondary resonance level of the brain tissue. The third ventricle was used as an 
echo chamber, and the ultrasound signals were digitally processed to obtain a digital high-resolution echopulsogram.[55] The echopulsogram represented ICP waves obtained invasively. Michaeli et al. [55] compared the TRA derived ICP with invasive ICP measurements in 40 patients and a high correlation $(r=0.99)$ was seen. They concluded that TRA allowed for accurate noninvasive ICP monitoring.[ㄷ5]

Based on Michaeli et al's[55] conclusions, TRA seemed promising for the monitoring of ICP noninvasively, however, we have been unable to find any other study that can support their findings.

Tonometry A tonometer is a device used to measure the intraocular pressure (IOP). Several studies have been carried out to test the correlation between IOP and ICP.[므, 71, 75] Sajjadi et al.[71] tested the hypothesis of a positive correlation between the two values in 50 patients. The Schiotz tonometer was used to measure IOP and a lumbar puncture was performed for ICP. They reached the conclusion that there was in fact a positive correlation between IOP and ICP $(P<0.001, r=0.955)$. [71] They also stated that the correlation was independent of factors such as BMI, age, and type of disease.[71]

Czarnik et al.[18] also conducted a similar study using the Schiotz tonometer for IOP and an intraparenchymal sensor for the ICP. Their study was conducted on 22 patients, and they found that a positive correlation between IOP and ICP was seen in only 2 patients. Therefore, they concluded that tonometry would not be a reliable method to assess ICP.

Most recent studies $[\underline{36}, \underline{52}, \underline{83}]$ have similar conclusions as Czarnik et al.[18] Golan et al.[하] concluded that the tonometer was not an effective device for screening ICP due to its poor specificity and sensitivity. Li et al.[52] verified the positive correlation between ICP and IOP $(r=0.32, P<0.001)$ but advised caution against using the tonometer for ICP assessment as they did not find significant correlations in the past studies that they quoted in their paper. Most recently, Tian et al. [으] found a significant positive correlation between ICP and IOP in patients with meningiomas $(46.7 \%$ of patients had a very significant correlation, $P<0.01,13.3 \%$ had a significant correlation, $P<0.05)$ but stated that changes in IOP could not always monitor the dynamic changes in ICP.

Acoustoelasticity The acoustoelastic effect describes the effect of a steady stress state on the sound velocities of an elastic material. In 2013, Wu et al.[92] proposed a novel experimental model based on the acoustoelasticity of ultrasound for the long-term, online, real-time, noninvasive monitoring of ICP. Their model was designed on the basis of consistent stress being present on the brain, such as in a closed container. Transducers were placed on the left and right and via conversion of electrical to mechanical energy and back to electrical energy, values of ICP were recorded.[92] A phase locked loop module was another component of the design; it was used to measure phase differences between signals, which were necessary for an embedded computer to evaluate the IC $P$ values. Polymethyl methacrylate was used to simulate the skull, and hydrogel was used to simulate the brain tissue in their experimental model.[92] A numerical solution for the acoustoelasticity theory was derived using a calculation based on a finite-element method. Calculated and experimental values were compared, and showed a maximum error of 5\%; the effectiveness of their model was verified, and the method was suggested for use in animal experimentation and clinical research.[92] A thorough literature search failed to identify any further studies conducted to evaluate this modality further.

Distortion-product otoacoustic emissions The CSF is continuous with the perilymphatic space, and therefore, changes in ICP can be transmitted to the middle ear.[22, $\underline{85}$ ] Increased ICP causes an increased stiffness of the annular ligament, and hence, increased pressure on the stapes, which in turn affects sound transmission. $[\underline{8}, \underline{12}, \underline{13}, \underline{22}, \underline{85}]$ Otoacoustic emissions (OAE) are generated via oscillations of the endo and perilymph, and have been shown to be sensitive to ICP changes. $[\underline{9}, \underline{13}, \underline{23}, \underline{29}, \underline{37}, \underline{59}, \underline{72}]$ Distortion product otoacoustic emission (DPOAE) is a type of otoacoustic emission that is often used for the assessment of middle ear function, $[\underline{8}, \underline{22}]$ and has been tested for noninvasive ICP monitoring. 
In 2006, Voss et al.[6] summarized prior work on the relationship between DPOAE and ICP changes; individuals who were in circumstances that would alter ICP also showed alterations in OAE magnitude and phase angles that were most pronounced at low frequencies $(<2000 \mathrm{~Hz})$, none of the reports however found how the two relate.[12, $\underline{13}, \underline{29}]$ Voss et al.[6] $]$ worked on the hypothesis that postural changes affect ICP, and compared postural changes and ICP, to DPOAE measurements in healthy normal-hearing individuals on a tilting-table. Their results showed that the predicted difference in DPOAE magnitudes, for frequencies $<1500 \mathrm{~Hz}$, between upright and $-45^{\circ}$ were highly significant $(P$ $<0.01)$, and were also highly significant for frequencies up to $1000 \mathrm{~Hz}$ between $90^{\circ}$ and $-30^{\circ}(P<$ 0.01). They concluded that DPOAE magnitudes, for frequencies between $750 \mathrm{~Hz}$ and $1500 \mathrm{~Hz}$, changed with posture systematically, and that this method could be assessed further for its use in monitoring ICP. In another study, Voss et al.[ㄷ] ] studied the effect of postural changes, and once again ICP, on DPOAE magnitude, angle, and power reflectance. All three measurements were made at two postural positions (upright and $-45^{\circ}$ to the horizontal). They found that the DPOAE magnitude changes were significant (median $P<0.01$ ) at frequencies between 600 and $1500 \mathrm{~Hz}$, the DPOAE angle changes were significant (median $P<0.01$ ) for frequencies between 600 and $3000 \mathrm{~Hz}$, and the power of reflectance changes were significant $(P<0.01)$ for frequencies below about $1000 \mathrm{~Hz}$. They used the repeated measures regression model, which predicted significant changes $(P<0.001)$ for all three measurements up to $2016 \mathrm{~Hz}$. They concluded that DPOAE magnitudes, angles, and power of reflectance all change with posture, and hence with ICP.

In 2012, Sakka et al.[72] measured DPOAE phase recordings in patients undergoing infusion tests for diagnosing chronic hydrocephalus. During the tests, 6 patients underwent continuous CSF pressure monitoring via an external transducer connected to a lumbar catheter. They confirmed a linear correlation between DPOAE phase shift and ICP variation in these patients; approximately $10^{\circ}$ DPOAE phase shift to $5 \mathrm{~cm} \mathrm{H}_{2} \mathrm{O}$ ICP change. However, their study did not compare actual ICP values to DPOAE measurements and each patient required calibration to relate the initial DPOAE to ICP. Bershad et al.[] just recently published their results from comparing ICP to DPOAEs on the basis of the hypothesis that DPOAE magnitudes increase, and phase angles decrease, with decrease in ICP. They measured opening and closing pressures during lumbar punctures, and simultaneously measured DPOAE magnitudes and phase angles. The measured ICP changes were grouped as small $(<4 \mathrm{mmHg})$, medium $(5-11 \mathrm{mmHg})$, and large $(\geq 15 \mathrm{mmHg})$. They found a significant ( $95 \%$ confidence interval, did not include 0) difference in DPOAE magnitude and angle, at $2000 \mathrm{~Hz}$, but only for the group that exhibited large ICP changes. They concluded that decreases in ICP of at least $15 \mathrm{mmHg}$ result in systematic changes in DPOAE magnitudes and angles, and their results were consistent with previous studies relating postural changes, ICP, and DPOAE measurements. Despite its limitations, it was the first study to prove that DPOAEs are directly affected by ICP variations. In conclusion, DPOAE measurements can possibly be an effective tool in noninvasive monitoring of ICP. However, more studies such as the one by Bershad et al.[]] need to be conducted on a much larger scale to determine the relationship more definitively.

Transcranial Doppler In 1982, Aaslid et al.[1] introduced transcranial Doppler (TCD) for monitoring cerebral hemodynamics. The use of TCD has been associated in several scenarios, such as the detection of vasospasm and cerebral embolization, cerebrovascular autoregulation, detection of cerebral circulatory arrest, traumatic brain injury, circulatory arrest, and monitoring of ICP.[누,,$\underline{70}]$ Klingelhofer et al. $[\underline{46}, \underline{47]}$ were the first to describe a relationship between ICP and TCD-derived flow velocities. They correlated an increasing ICP with decreasing TCD-derived flow velocities and an increase in Pourcelot index or resistance index (RI), where RI = (systolic flow velocity - diastolic flow velocity)/(systolic flow velocity).[49] At present, however, the Gosling pulsatility index (PI), PI = (systolic flow velocity - diastolic flow velocity)/(mean flow velocity),[르] is the most commonly used

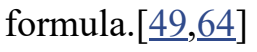


The middle cerebral artery (MCA) is most commonly used for TCD measurements. Both the MCAs are insonated, after which the blood flow is directed towards the probe.[11] The Doppler instrument operates at $2 \mathrm{MHz}[\underline{11}, \underline{49}]$ and the angle and position of the exposure area can be adjusted to enable the highest quality signal. Bilateral tracings of flow velocity are recorded, and necessary calculations are made to obtain the PI.[11] The main advantage of PI is that, since it is a ratio, it is not affected by the angle of insonation. $[11, \underline{49}]$

In a recent review on the role of the TCD,[11] it was highlighted that the correlation between PI and ICP is still controversial. The strength of the correlation has also showed variations in many studies. $[\underline{6}, \underline{7}, \underline{10}, \underline{21}, \underline{89}, \underline{94]}$ Riva et al.[리] even questioned the usefulness of the TCD technique. In 2014 Wakerley et al.[90] studied TCD as a modality to monitor ICP noninvasively. They conducted their study on 78 patients, obtaining the TCD spectra from either MCA using a $2 \mathrm{MHz}$ transducer and then after 5 minutes measuring cerebrospinal fluid pressures invasively via a lumbar puncture (LP).[90] They found that $\mathrm{PI} \geq 1.26$ reliably predicted CSF- $\mathrm{p} \geq 20 \mathrm{cmH}_{2} \mathrm{O}$ with sensitivity $=81.1 \%$, specificity $=$ $96.3 \%$, positive predictive value $=88.1 \%$, and negative predictive value $=90.1 \%$. [900] They concluded that the TCD-derived PI could play an important role as a monitoring device. Other studies showed that TCD could be used to estimate ICP, but had doubts regarding its use in clinical scenarios due to its reliability.[묵, $\underline{71}, \underline{25}, \underline{38}, \underline{40}, \underline{58}, \underline{66}, \underline{87}]$

In 2004, Bellner et al.[7] conducted a prospective study to evaluate the relationship between ICP and TCD-derived PI. TCD recordings were taken daily and the ICP was monitored using an intraventricular catheter. They found a significant correlation between the values $(P<0.0001, r=0.938$ for the formula $\mathrm{ICP}=10.93 \times \mathrm{PI}-1.28$ ), and concluded that independent of the type of pathology the two values had a strong correlation.[7] Since then, however, no other group has made a statistical prediction; in their review article, Kristiansson et al.[49] also mentioned that the use of PI in clinical practice depends on evidence of how well PI-derived ICP reflects the actual ICP.

In 2009, Figaji et al.[25] investigated the association of TCD-derived PI and ICP in children with severe traumatic brain injury (TBI). In their study, 275 TCD studies were conducted on 34 children, all of whom had invasive ICP monitors placed beforehand. They found a weak relationship between the mean values of ICP and PI $(P=0.04, r=0.36)$, and PI threshold of 1 for examining an ICP threshold of $20 \mathrm{mmHg}$ had a sensitivity of $25 \%$ and specificity of $88 \%$.[25] They, therefore, concluded that the absolute value of PI was not reliable as an indicator for ICP in children with severe TBI.[25]

Raguskas et al.[65] evaluated the accuracy and precision of a two-depth high resolution TCD device for measuring ICP. The intracranial and extracranial segments of the ophthalmic artery were used. The intracranial segment represented ICP and the extracranial segment represented pressure applied to the orbit. It was found that when the pressures balanced, the blood flow parameters were the same. The ICP was also measured invasively to compare the recordings. Seventy-two simultaneously paired recordings were analyzed which showed good accuracy (mean systematic error $=0.12 \mathrm{mmHg}$, confidence level $=0.98)$ and high precision ( for the two-depth TCD.

As mentioned by Kristiansson et al.,[49] it is important to derive an equation that reliably describes the relationship between ICP and TCD findings. To date, the PI has not shown absolute ICP values but only reveals changes in ICP There are also no standardized cut-off values for PI between studies.[49] Therefore, TCD seems to be a promising modality for the non-invasive monitoring of ICP, however, it cannot replace invasive ICP monitoring.

Electroencephalogram Initially studies were conducted to assess the role of continuous electroencephalogram (EEG) monitoring for the prognosis of TBI.[로,,$\underline{51}$ ] Lescot et al.[1] studied the relationship between Lundberg waves (repetitive ICP changes at a frequency of 0.5-2 waves per minute) and EEG fluctuations in 6 brain injured patients. They concluded that changes in cerebral 
electrical activity could be attributed to increasing cerebral metabolic rate of oxygen, which increases cerebral blood flow, therefore leading to an increase in ICP due to a change in blood volume. However, there was no assessment of the role of EEG for monitoring ICP directly. In 2012, Amantini et al.[2] discussed the role of neurophysiological monitoring in the intensive care unit (ICU). One of their favoring points for the use of EEG in the ICU was that it could be used to interpret and manage ICP trends, as neurophysiologic alterations were found to precede ICP increases. They did not, however, state any correlation between EEG findings and ICP values.

Chen et al.[15] investigated the possibility of using EEG power spectrum analysis to monitor ICP noninvasively on 62 patients with CNS disorders. EEGs were recorded and a self-designed software was used to automatically calculate the pressure index (PI), while lumbar punctures were obtained to measure the ICP. A significant negative correlation was found between PI and ICP $(r=-0.849, P<$ 0.01 ), and the authors concluded that specific parameters from the EEG power spectrum might reflect the ICP. Further studies may be warranted to assess the use of EEG power spectrum in ICP monitoring.

Near-infrared spectroscopy Near-infrared spectroscopy (NIRS) has also been indicated to monitor TBI patients. It can detect changes in cerebral blood volume (CBV), brain tissue oxygenation, and cerebral blood flow (CBF).[33] At the near infrared spectrum of light there is low absorptivity that allows deep tissue penetration. The variability of the absorptivity helps quantify the detected changes in the concentrations of deoxyhemoglobin and oxyhemoglobin.

In 1995, Kirkpatrick et al.[45] investigated the potential use of NIRS in 14 patients with closed head injuries. The purpose of their study was to compare the ability of NIRS and jugular venous saturation $\left(\mathrm{SjO}_{2}\right)$ monitors in detecting cerebral deoxygenation changes. They monitored ICP, cerebral perfusion pressure (CPP), peripheral oxygenation saturation, jugular venous saturation, and NIRS derived changes in oxy- and deoxyhemoglobin. They reviewed 886 hours of continuous monitoring, of which 376 hours were analyzed. They found that NIRS detected changes in $97 \%$ of the hemodynamic changes, whereas $\mathrm{SjO}_{2}$ monitor detected 53\%. Every NIRS detected change was accompanied by changes in ICP, CPP, and relative CBF changes. They concluded that, even though the technique of NIRS use in the adult brain was not completely understood, there were clear signal changes associated with variations in ICP, CPP, and CBF. Their study, however, did not correlate NIRS findings with actual ICP readings.

Wagner et al.[88] used NIRS to monitor the changes in cerebral hemoglobin saturation and content in six children in coma due to severe encephalopathy. Because CBV is an important determinant of ICP, it was stated that acute changes in CBV due to vessel diameter should induce acute changes in ICP.[요] They tested cerebrovascular detection by NIRS by investigating whether ICP changes would correlate with changes in NIRS signals. ICP was monitored using an epidural or an intraparenchymal device. A significant correlation between cerebral hemoglobin saturation and changes in ICP was found, with a correlation coefficient of $0.82(P<.0001)$. A weaker correlation was also found between cerebral hemoglobin content and changes in ICP, with a correlation coefficient of $0.58(P=0.0006)$.[요 $]$ However, similarly to the previous study, their study was not conducted to evaluate the correlation between quantitative ICP readings and NIRS findings.

A recent review article[49] also discussed the potential use of NIRS for the noninvasive monitoring of ICP and concluded that NIRS shows to have promise for the assessment of patients with traumatic brain injury, however, it cannot currently be used to estimate absolute ICP readings.

Pupillometry In 1983, Marshall et al.[54] observed the pupillary changes of patients requiring continuous ICP monitoring. They recorded the ICP when shape of the pupil became oval, and then tracked the pupillary and ICP changes in response to intracranial hypertension therapy to find an association between pupillary shape and changes in ICP.[54] Over a 2-year period, 14 instances of an oval pupil in patients with raised ICP were observed. Almost every instance showed a return to normal 
pupil size when ICP returned to normal values.[54] They concluded that the oval pupil represented a stage between the normal pupil and the fixed unreactive pupil of high ICP, and therefore, suggested that any patient with an oval pupil should receive immediate management.[54] Their study, however, did not indicate any specific ICP values to pupillary shape changes. The author's also discussed the possibility of detecting elevated ICP before it occurs by measurements of pupillary sphincter function. $[\underline{54}, \underline{82}]$ Since then, many studies have been conducted to assess pupillary changes in severely ill patients to assess their outcome.[르]

In 2003, Taylor et al.[ㄹ] investigated the use of a new hand-held point-and-shoot pupillometer (ForSite; NeurOptics) to quantitatively assess pupillary function. A total of 404 volunteers were enrolled, of which 26 had head injuries and their ICP was continuously monitored. The purpose of their study was to conclude whether the device could reliably provide accurate assessment of patterns of pupillary responsiveness, not to find an association between pupillary changes and ICP.[르 They did, however, establish that pupillary changes could reveal subtle changes in ICP; $[\underline{28}, \underline{82}]$ it was found that pupillary constriction velocity was quite sensitive to raised ICP, and a reduction in pupillary size by $10 \%$ was always associated with an ICP $>20 \mathrm{mmHg}$.[르 It is important to note that there was no correlation of specific pupillary size to actual ICP values, however, they concluded that there was a strong relationship between mass effect, ICP $>20 \mathrm{mmHg}$, and a reduction in constriction velocity. $[\underline{28}, \underline{82}]$

More recently, Chen et al.[16] introduced the neurological pupillary index (NPi) and studied pupillary reactivity as an early indicator of increased ICP. A total of 134 patients were enrolled, the NeurOptic's pupillometer was used for pupillary examination, and the ICP was continuously monitored. The pupillometer can accurately grade a pupil's response to light, using an algorithm incorporating pupillary light reflex variables.[17] These scores are called NPi and are set on a scale from 0 to 5, scores falling below. Their results showed that normal pupil reactivity had an average ICP of $19.6 \mathrm{mmHg}$, abnormal pupillary reactivity had an average ICP peak of $30.5 \mathrm{mmHg}$, and nonreactive pupils had the highest ICP peaks (mean $=33.8 \mathrm{mmHg}, P=0.0046)$. [16] They also found that pupil abnormalities occurred, on average, 15.9 hours before an increase in ICP. They concluded that an inverse trend between decreasing pupillary response and increasing ICP was identified, and quantitative measurement of NPi may be useful for early management of increased ICP.[16] However, there still is no direct correlation between NPi and actual values of ICP, and it cannot be used to continuously monitor ICP.

In conclusion, pupillometry is a useful tool for screening patients with possibly increased ICP, however, because conclusive ICP values cannot be detected by this modality, it cannot be suggested for continuous ICP monitoring.

Intracranial pressure is elevated in several clinical settings, especially TBI and stroke. The cumulative incidence of these two conditions is approximately $0.6 \%$ in developed countries, and their cumulative mortality rate is $30-50 \%$, both of which indicate a major burden of disease.[23] Elevated ICP is primarily responsible for secondary brain ischemia after either condition, and therefore, is responsible for the functional disability many of these patients suffer. In such instances, it has been suggested that controlling the intracranial hypertension is the standard of care in the management these patients. Therefore, monitoring ICP through a reliable, invasive technique is used to evaluate the progress in patient management, help formulate treatment strategies, and in cases of intraventricular catheters, may also help in relieving the intracranial hypertension through CSF drainage. For routine use, however, we feel that noninvasive ICP monitoring if developed to be sensitive enough, is the future of neurointensive monitoring. The ideal ICP monitoring modality in our opinion should be safe, reliable, cost effective, easily available, non-operator dependent, and noninvasive. 
We feel that, even though at the moment there is no modality of noninvasive ICP monitoring that can replace invasive ICP monitoring, noninvasive monitoring may still be useful in centers where invasive modalities are not available, such as in developing countries, or even in developed countries as a screening tool to decide, which patients will require invasive monitoring. Invasive ICP monitoring is expensive and requires the availability of a neurosurgeon, both of which are very difficult to acquire in an under resourced regions and also at many trauma centers.[를

Of all the different modalities for noninvasive monitoring that we have studied, the authors are of the opinion that two stand out. These are measuring the ONSD and pupillometry. Both modalities are reliable, efficient, affordable, and most importantly, easy to learn. Several studies are ongoing to evaluate their utility in greater depth. Pupillometry, we feel will gain widespread popularity, especially after the introduction of a commercially available, easy to use, nonoperator dependent, electronic pupillometer. Radiology, specifically repeat CT scans for monitoring elevated ICP, has always been, and continues to be an important tool for clinicians, although it was beyond the scope of this paper.

Invasive ICP monitoring via a ventricular catheter remains the gold standard, however, there are many areas around the world and several situations where this modality, or other means of invasive ICP monitoring cannot be utilized. Noninvasive modalities provide a useful alternative under such circumstances. Several modalities are available, and even though the ideal modality is yet to be introduced, a number of techniques can be employed. Of these, ONSD and pupillometry may be two modalities to look out for in the future.

\section{Financial support and sponsorship}

Nil.

\section{Conflicts of interest}

There are no conflicts of interest.

Footnotes

Go to:

http://surgicalneurologyint.com/Noninvasive-monitoring-intracranial-pressure-A-review-of-available-modalities/

\section{REFERENCES}

Go to:

1. Aaslid R, Markwalder TM, Nornes H. Noninvasive transcranial Doppler ultrasound recording of flow velocity in basal cerebral arteries. J Neurosurg. 1982;57:769-74. [PubMed]

2. Amantini A, Carrai R, Lori S, Peris A, Amadori A, Pinto F, et al. Neurophysiological monitoring in adult and pediatric intensive care. Minerva Anestesiol. 2012;78:1067-75. [PubMed]

3. Amantini A, Fossi S, Grippo A, Innocenti P, Amadori A, Bucciardini L, et al. Continuous EEG-SEP monitoring in severe brain injury. Neurophysiol Clin. 2009;39:85-93. [PubMed]

4. Ballantyne S, O'Neill G, Hamilton R, Hollman A. Observer variation in the sonographic measurement of optic nerve sheath diameter in normal adults. Eur J Ultrasound. 2002;15:145-9. [PubMed]

5. Baurmann M. Über die Entstehung und klinische Bedeutung des Netzhautvenenpulses. Dtsch Ophthalmol Ges. 1925;45:53-9.

6. Behrens A, Lenfeldt N, Ambarki K, Malm J, Eklund A, Koskinen LO. Transcranial Doppler pulsatility index: Not an accurate method to assess intracranial pressure. Neurosurgery. 2010;66:1050- 


\section{7. [PubMed]}

7. Bellner J, Romner B, Reinstrup P, Kristiansson KA, Ryding E, Brandt L. Transcranial Doppler sonography pulsatility index (PI) reflects intracranial pressure (ICP) Surg Neurol. 2004;62:45-51. [PubMed]

8. Bershad EM, Urfy MZ, Pechacek A, McGrath M, Calvillo E, Horton NJ, et al. Intracranial pressure modulates distortion product otoacoustic emissions: A proof-of-principle study. Neurosurgery.

2014;75:445-54. [PubMed]

9. Bohmer A. Hydrostatic pressure in the inner ear fluid compartments and its effects on inner ear function. Acta Otolaryngol Suppl. 1993;507:3-24. [ubMed]

10. Bouzat P, Francony G, Declety P, Genty C, Kaddour A, Bessou P, et al. Transcranial Doppler to screen on admission patients with mild to moderate traumatic brain injury. Neurosurgery.

2011;68:1603-10. [ubMed]

11. Bouzat P, Oddo M, Payen JF. Transcranial Doppler after traumatic brain injury: Is there a role? Curr Opiion in Crit Care. 2014;20:153-60. [PubMed]

12. Buki B, Avan P, Lemaire JJ, Dordain M, Chazal J, Ribari O. Otoacoustic emissions: A new tool for monitoring intracranial pressure changes through stapes displacements. Hear Res. 1996;94:125-39. [PubMed]

13. Buki B, de Kleine E, Wit HP, Avan P. Detection of intracochlear and intracranial pressure changes with otoacoustic emissions: A gerbil model. Hear Res. 2002;167:180-91. [릴ed]

14. Bunegin L, Albin MS, Rauschhuber R, Marlin AE. Intracranial pressure measurement from the anterior fontanelle utilizing a pneumoelectronic switch. Neurosurgery. 1987;20:726-731. [PubMed]

15. Chen H, Wang J, Mao S, Dong W, Yang H. A new method of intracranial pressure monitoring by EEG power spectrum analysis. Can J Neurol Sci. 2012;39:483-7. [PubMed]

16. Chen JW, Gombart ZJ, Rogers S, Gardiner SK, Cecil S, Bullock RM. Pupillary reactivity as an early indicator of increased intracranial pressure: The introduction of the Neurological Pupil index. Surg Neurol Int. 2011;2:82. [PMC free article] [PubMed]

17. Chen JW, Vakil-Gilani K, Williamson KL, Cecil S. Infrared pupillometry, the Neurological Pupil index and unilateral pupillary dilation after traumatic brain injury: Implications for treatment paradigms. SpringerPlus. 2014;3:548. [MC free article] [PubMed]

18. Czarnik T, Gawda R, Latka D, Kolodziej W, Sznajd-Weron K, Weron R. Noninvasive measurement of intracranial pressure: Is it possible? J Trauma. 2007;62:207-11. [PubMed]

19. de Jong DA, Berfelo MW, de Lange SA, Maas AI. Epidural pressure monitoring with the so-called Rotterdam transducer. Further in vivo results. Acta Neurochir. 1979;45:301-9. [PubMed]

20. De Jong DA, Maas AI, v d Voort E. Non-invasive intracranial pressure monitoring. A technique for reproducible fontanelle pressure measurements. Z Kinderchir. 1984;39:274-6. [ubMed]

21. de Riva N, Budohoski KP, Smielewski P, Kasprowicz M, Zweifel C, Steiner LA, et al. Transcranial Doppler pulsatility index: What it is and what it isn't. Neurocritical Care. 2012;17:58-66. [PubMed]

22. Deppe C, Kummer P, Gurkov R, Olzowy B. Influence of the individual DPOAE growth behavior on DPOAE level variations caused by conductive hearing loss and elevated intracranial pressure. Ear Hear. 2013;34:122-31. [PubMed] 
23. Djorfe Popovic MK, Stefan Lee. Noninvasive Monitoring of Intracranial Pressure. Recent Patents on Biomedical Engineering. 2009;2:165-79.

24. Eide PK, Bakken A. The baseline pressure of intracranial pressure (ICP) sensors can be altered by electrostatic discharges. Biomed Eng Online. 2011;10:75. [MC free article] [PubMed]

25. Figaji AA, Zwane E, Fieggen AG, Siesjo P, Peter JC. Transcranial Doppler pulsatility index is not a reliable indicator of intracranial pressure in children with severe traumatic brain injury. Surg Neurol. 2009;72:389-94. [PubMed]

26. Firsching R, Muller C, Pauli SU, Voellger B, Rohl FW, Behrens-Baumann W. Noninvasive assessment of intracranial pressure with venous ophthalmodynamometry. Clinical article. J Neurosurg. 2011;115:371-4. [PubMed]

27. Firsching R, Schutze M, Motschmann M, Behrens-Baumann W. Venous opthalmodynamometry: A noninvasive method for assessment of intracranial pressure. J Neurosurg. 2000;93:33-6. [PubMed]

28. Fountas KN, Kapsalaki EZ, Machinis TG, Boev AN, Robinson JS, Troup EC. Clinical implications of quantitative infrared pupillometry in neurosurgical patients. Neurocrit Care. 2006;5:55-60. [PubMed]

29. Frank AM, Alexiou C, Hulin P, Janssen T, Arnold W, Trappe AE. Non-invasive measurement of intracranial pressure changes by otoacoustic emissions (OAEs)--a report of preliminary data. Zentralbl Neurochir. 2000;61:177-80. [PubMed]

30. Gaihede M, Felding JU, Elbrônd O. Biomechanical Characteristics of the Middle Ear System Measured by a New Method: III: Comparisons with Tympanometric Measurements. Acta Otolaryngol. 1995;115:522-7. [PubMed]

31. Geeraerts T, Duranteau J, Benhamou D. Ocular sonography in patients with raised intracranial pressure: The papilloedema revisited. Crit Care. 2008;12:150. [PMC free article] [PubMed]

32. Geeraerts T, Launey Y, Martin L, Pottecher J, Vigué B, Duranteau J, et al. Ultrasonography of the optic nerve sheath may be useful for detecting raised intracranial pressure after severe brain injury. Intensive Care Med. 2007;33:1704-11. [PubMed]

33. Ghosh A, Elwell C, Smith M. Review article: Cerebral near-infrared spectroscopy in adults: A work in progress. Anesth Analg. 2012;115(6):1373-1383. [ubMed]

34. Gilland O. Normal cerebrospinal-fluid pressure. N Eng J Med. 1969;280:904-5. [PubMed]

35. Gilland O, Tourtellotte WW, O'Tauma L, Henderson WG. Normal cerebrospinal fluid pressure. J Neurosurg. 1974;40:587-93. [PubMed]

36. Golan S, Kurtz S, Mezad-Koursh D, Waisbourd M, Kesler A, Halpern P. Poor correlation between intracranial pressure and intraocular pressure by hand-held tonometry. Clin Ophthalmol. 2013;7:1083. [PMC free article] [PubMed]

37. Gopen Q, Rosowski JJ, Merchant SN. Anatomy of the normal human cochlear aqueduct with functional implications. Hear Res. 1997;107:9-22. [PubMed]

38. Gura M, Silav G, Isik N, Elmaci I. Noninvasive estimation of cerebral perfusion pressure with transcranial Doppler ultrasonography in traumatic brain injury. Turkish Neurosurg. 2012;22:411-5. [PubMed]

39. Hayreh SS. Pathogenesis of oedema of the optic disc (pappiloedema).A preliminary report. Br J Ophthalmol. 1964;48:522-43. [Mㅡ free article] [PubMed] 
40. Homburg AM, Jakobsen M, Enevoldsen E. Transcranial Doppler recordings in raised intracranial pressure. Acta Neurol Scand. 1993;87:488-93. [PubMed]

41. Horbar JD, Yeager S, Philip AG, Lucey JF. Effect of application force on noninvasive measurements of intracranial pressure. Pediatrics. 1980;66:455-7. [PubMed]

42. Killer HE, Laeng HR, Flammer J, Groscurth P. Architecture of arachnoid trabeculae, pillars, and septa in the subarachnoid space of the human optic nerve: Anatomy and clinical considerations. Br J Ophthalmol. 2003;87:777-81. [PMC free article] [PubMed]

43. Kim YK, Seo H, Yu J, Hwang GS. Noninvasive estimation of raised intracranial pressure using ocular ultrasonography in liver transplant recipients with acute liver failure -A report of two cases. Korean J Anesthesiol. 2013;64:451-5. [M free article] [라Med]

44. Kimberly HH, Shah S, Marill K, Noble V. Correlation of optic nerve sheath diameter with direct measurement of intracranial pressure. Acad Emerg Med. 2008;15:201-4. [PubMed]

45. Kirkpatrick PJ, Smielewski P, Czosnyka M, Menon DK, Pickard JD. Near-infrared spectroscopy use in patients with head injury. J Neurosurg. 1995;83:963-70. [PubMed]

46. Klingelhöfer J, Conrad B, Benecke R, Sander D. Intracranial flow patterns at increasing intracranial pressure. Klin Wochenschr. 1987;65:542-5. [ubMed]

47. Klingelhöfer J, Conrad B, Benecke R, Sander D, Markakis E. Evaluation of intracranial pressure from transcranial Doppler studies in cerebral disease. J Neurol. 1988;235:159-62. [PubMed]

48. Krishnamoorthy V, Beckmann K, Mueller M, Sharma D, Vavilala MS. Perioperative estimation of the intracranial pressure using the optic nerve sheath diameter during liver transplantation. Liver Transpl. 2013;19:246-9. [PubMed]

49. Kristiansson H, Nissborg E, Bartek J, Jr, Andresen M, Reinstrup P, Romner B. Measuring elevated intracranial pressure through noninvasive methods: A review of the literature. J Neurosurg Anesthesiol. 2013;25:372-85. [PubMed]

50. Lashutka MK, Chandra A, Murray HN, Phillips GS, Hiestand BC. The relationship of intraocular pressure to intracranial pressure. Ann Emerg Med. 2004;43:585-91. [PubMed]

51. Lescot T, Naccache L, Bonnet MP, Abdennour L, Coriat P, Puybasset L. The relationship of intracranial pressure Lundberg waves to electroencephalograph fluctuations in patients with severe head trauma. Acta Neurochir. 2005;147:125-9. [PubMed]

52. Li Z, Yang Y, Lu Y, Liu D, Xu E, Jia J, et al. Intraocular pressure vs intracranial pressure in disease conditions: A prospective cohort study (Beijing iCOP study) BMC Neurol. 2012;12:66. [PMC free article] [ubMed]

53. Marchbanks RJ. Measurement of tympanic membrane displacement arising from aural cardiovascular activity, swallowing, and intra-aural muscle reflex. Acta Otolaryngol. 1984;98:119-29. [PubMed]

54. Marshall LF, Barba D, Toole BM, Bowers SA. The oval pupil: Clinical significance and relationship to intracranial hypertension. J Neurosurg. 1983;58:566-8. [ubMed]

55. Michaeli D, Rappaport ZH. Tissue resonance analysis; a novel method for noninvasive monitoring of intracranial pressure. Technical note. J Neurosurg. 2002;96:1132-7. [PubMed]

56. Miller MT, Pasquale M, Kurek S, White J, Martin P, Bannon K, et al. Initial head computed tomographic scan characteristics have a linear relationship with initial intracranial pressure after trauma. J Trauma. 2004;56:967-72. [PubMed] 
57. Mokri B. The Monro-Kellie hypothesis: Applications in CSF volume depletion. Neurology. 2001;56:1746-8. [PubMed]

58. Moreno JA, Mesalles E, Gener J, Tomasa A, Ley A, Roca J, et al. Evaluating the outcome of severe head injury with transcranial Doppler ultrasonography. Neurosurg Focus. 2000;8:1-7. [PubMed]

59. Murakami S, Gyo K, Goode RL. Effect of increased inner ear pressure on middle ear mechanics. Otolaryngol Head Neck Surg. 1998;118:703-8. [PubMed]

60. Overweg-Plandsoen WCG. Anterior fontanelle pressure monitoring in infants [Ph.D. thesis]: Erasmus University Rotterdam. 1990

61. Pitlyk PJ, Piantanida TP, Ploeger DW. Noninvasive intracranial pressure monitoring. Neurosurgery. 1985;17:581-4. [ubMed]

62. Plandsoen WC, de Jong DA, Maas AI, Stroink H, Avezaat CJ. Fontanelle pressure monitoring in infants with the Rotterdam Teletransducer: A reliable technique. Med Prog Technol. 1987;13:21-7. [PubMed]

63. Querfurth HW, Arms SW, Lichy CM, Irwin WT, Steiner T. Prediction of intracranial pressure from noninvasive transocular venous and arterial hemodynamic measurements: A pilot study. Neurocrit Care. 2004;1:183-94. [PubMed]

64. Raboel PH, Bartek J, Jr, Andresen M, Bellander BM, Romner B. Intracranial Pressure Monitoring: Invasive versus Non-Invasive Methods-A Review. Crit Care Res Pract 2012. 2012:950393. [PMC free article] [․ㅏbMed]

65. Ragauskas A, Matijosaitis V, Zakelis R, Petrikonis K, Rastenyte D, Piper I, et al. Clinical assessment of noninvasive intracranial pressure absolute value measurement method. Neurology. 2012;78:1684-91. [PubMed]

66. Rainov N, Weise JB, Burkert W. Transcranial Doppler sonography in adult hydrocephalic patients. Neurosurg Rev. 2000;23:34-8. [PubMed]

67. Rajajee V, Vanaman M, Fletcher JJ, Jacobs TL. Optic nerve ultrasound for the detection of raised intracranial pressure. Neurocrit Care. 2011;15:506-15. [PubMed]

68. Raksin PB, Alperin N, Sivaramakrishnan A, Surapaneni S, Lichtor T. Noninvasive intracranial compliance and pressure based on dynamic magnetic resonance imaging of blood flow and cerebrospinal fluid flow: Review of principles, implementation, and other noninvasive approaches. Neurosurg Focus. 2003;14:e4. [ubMed]

69. Reid A, Marchbanks R, Burge D, Martin A, Bateman D, Pickard J, et al. The relationship between intracranial pressure and tympanic membrane displacement. Br J Audiol. 1990;24:123-9. [PubMed]

70. Rosenberg JB, Shiloh AL, Savel RH, Eisen LA. Non-invasive methods of estimating intracranial pressure. Neurocrit Care. 2011;15:599-608. [PubMed]

71. Sajjadi SA, Harirchian MH, Sheikhbahaei N, Mohebbi MR, Malekmadani MH, Saberi H. The relation between intracranial and intraocular pressures: Study of 50 patients. Ann Neurol.

2006;59:867-70. [PubMed]

72. Sakka L, Thalamy A, Giraudet F, Hassoun T, Avan P, Chazal J. Electrophysiological monitoring of cochlear function as a non-invasive method to assess intracranial pressure variations. Acta Neurochir Suppl. 2012;114:131-4. [PubMed]

73. Salmon JH, Hajjar W, Bada HS. The fontogram: A noninvasive intracranial pressure monitor. Pediatrics. 1977;60:721-5. [ubMed] 
74. Samuel M, Burge DM, Marchbanks RJ. Tympanic membrane displacement testing in regular assessment of intracranial pressure in eight children with shunted hydrocephalus. J Neurosurg. 1998;88:983-95. [PubMed]

75. Sheeran P, Bland J, Hall G. Intraocular pressure changes and alterations in intracranial pressure. Lancet. 2000;355:899. [릴ed]

76. Smith M. Monitoring intracranial pressure in traumatic brain injury. Anesth Analg. 2008;106:2408. [PubMed]

77. Soldatos T, Chatzimichail K, Papathanasiou M, Gouliamos A. Optic nerve sonography: A new window for the non-invasive evaluation of intracranial pressure in brain injury. Emerg Med J. 2009;26:630-4. [ubMed]

78. Soldatos T, Karakitsos D, Chatzimichail K, Papathanasiou M, Gouliamos A, Karabinis A. Optic nerve sonography in the diagnostic evaluation of adult brain injury. Crit Care. 2008;12:R67. [PMC free article] [PubMed]

79. Stettin E, Paulat K, Schulz C, Kunz U, Mauer UM. Noninvasive intracranial pressure measurement using infrasonic emissions from the tympanic membrane. J Clin Monit Comput. 2011;25:203-10. [PubMed]

80. Strumwasser A, Kwan RO, Yeung L, Miraflor E, Ereso A, Castro-Moure F, et al. Sonographic optic nerve sheath diameter as an estimate of intracranial pressure in adult trauma. J Surg Res.

2011;170:265-71. [PubMed]

81. Tayal VS, Neulander M, Norton HJ, Foster T, Saunders T, Blaivas M. Emergency department sonographic measurement of optic nerve sheath diameter to detect findings of increased intracranial pressure in adult head injury patients. Ann Emerg Med. 2007;49:508-14. [릴ed]

82. Taylor WR, Chen JW, Meltzer H, Gennarelli TA, Kelbch C, Knowlton S, et al. Quantitative pupillometry, a new technology: Normative data and preliminary observations in patients with acute head injury. Technical note. J Neurosurg. 2003;98:205-13. [ubMed]

83. Chunyu T, XP, Li N, Qin L, Tian Z. The Correlation between Intracranial Pressure and Intraocular Pressure after Brain Surgery. Int J Ophthalmol Eye Res. 2014;2:54-8.

84. Vidyasagar D, Raju TN. A simple noninvasive technique of measuring intracranial pressure in the newborn. Pediatrics. 1977;59:957-61. [PubMed]

85. Voss SE, Adegoke MF, Horton NJ, Sheth KN, Rosand J, Shera CA. Posture systematically alters ear-canal reflectance and DPOAE properties. Hear Res. 2010;263:43-51. [PMC free article] [PubMed]

86. Voss SE, Horton NJ, Tabucchi TH, Folowosele FO, Shera CA. Posture-induced changes in distortion-product otoacoustic emissions and the potential for noninvasive monitoring of changes in intracranial pressure. Neurocrit Care. 2006;4:251-7. [ubMed]

87. Voulgaris SG, Partheni M, Kaliora H, Haftouras N, Pessach IS, Polyzoidis KS. Early cerebral monitoring using the transcranial Doppler pulsatility index in patients with severe brain trauma. Med Sci Monit. 2005;11:CR49-52. [ubMed]

88. Wagner BP, Pfenninger J. Dynamic cerebral autoregulatory response to blood pressure rise measured by near-infrared spectroscopy and intracranial pressure. Crit Care Med. 2002;30:2014-21. [PubMed]

89. Wakerley B, Yohana K, Luen Teoh H, Tan CW, Chan BP, Sharma VK. Non-invasive intracranial pressure monitoring with transcranial Doppler in a patient with progressive cerebral venous sinus 
thrombosis. J Neuroimaging. 2014;24:302-4. [PubMed]

90. Wakerley BR, Kusuma Y, Yeo LL, Liang S, Kumar K, Sharma AK, et al. Usefulness of Transcranial Doppler-Derived Cerebral Hemodynamic Parameters in the Noninvasive Assessment of Intracranial Pressure. J Neuroimaging. 2015;25:111-6. [PubMed]

91. Wiegand C, Richards P. Measurement of intracranial pressure in children: A critical review of current methods. Dev Med Child Neurol. 2007;49:935-41. [PubMed]

92. Wu J, He W, Chen WM, Zhu L. Research on simulation and experiment of noninvasive intracranial pressure monitoring based on acoustoelasticity effects. Med Devices. 2013;6:123-31.

[PMC free article] [PubMed]

93. Xianfang Yue LW. Deformation of skull bone as intracranial pressure changing. African Journal of Biotechnology. 2009;8:745-50. ISSN 1684-5315.

94. Zweifel C, Czosnyka M, Carrera E, de Riva N, Pickard JD, Smielewski P. Reliability of the blood flow velocity pulsatility index for assessment of intracranial and cerebral perfusion pressures in headinjured patients. Neurosurgery. 2012;71:853-61. [PubMed]

Articles from Surgical Neurology International are provided here courtesy of Wolters Kluwer -- Medknow Publications 